\title{
Fast Non-Monte-Carlo Transient Noise Analysis for High-Precision Analog/RF Circuits by Stochastic Orthogonal Polynomials
}

\author{
Fang Gong \\ University of California, Los \\ Angeles \\ Electrical Engineering \\ Department \\ Los Angeles, CA 90095, US \\ gongfang@ucla.edu
}

\author{
$\mathrm{Hao} \mathrm{Yu}$ \\ Nanyang Technological \\ University \\ Electrical and Electronic \\ Engineering \\ haoyu@ntu.edu.sg
}

\author{
Lei He \\ University of California, Los \\ Angeles \\ Electrical Engineering \\ Department \\ Los Angeles, CA 90095, US \\ Ihe@ee.ucla.edu
}

\begin{abstract}
Stochastic device noise has been a significant challenge for highprecision analog/RF circuits, and it is particularly difficult to correctly include both white noise and flicker noise into the traditional transient verification flow with efficient numerical solution. In this paper, a Non-Monte-Carlo transient noise analysis is developed. Both white noise and flicker noise are considered in the Itô integral based stochastic differential algebraic equation (SDAE), which is further solved with one-time calculation of variance using the stochastic orthogonal polynomials (SoPs). This work is the first to provide SoP based SDAE solution with application in transient noise analysis. Experiments on a number of different analog circuits demonstrate that the proposed method is up to 488X faster than Monte Carlo method with a similar accuracy, and achieves on average $6.8 \mathrm{X}$ speedup over existing non-MonteCarlo approaches.
\end{abstract}

\section{INTRODUCTION}

Device noise is one of fundamental limits for circuit performance. Noise-related issues are particularly critical for high-precision circuits implemented at nanometer-scale with low voltages or high frequencies. For example, random device noise has a significant impact on nanometer CMOS PLL phase noise and jitter[1]. Note that the noise-sensitive analog/RF circuits such as ADCs and PLLs are the core components for bio-sensory and wireless communication systems. The device random noise is primarily composed of white noise (thermal and shot) and flicker noise. Thermal noise is broadband white noise that intensifies as temperature increases. In contrast, flicker noise is due to defects in semiconductor. The frequency at which the flicker-noise spectral density intersects the flat white-noise spectral density is called $1 / \mathrm{f}$ corner frequency. Both thermal and flick noise can be modeled inside the device model for the transistor. The primary challenge is to verify the transient noise behavior at circuit and system level with multiple transistors. Mainly due to the stochastic verification of device noise, the design for high-precision analog/RF components is usually time-consuming. The traditional SPICE-like verifica-

Permission to make digital or hard copies of all or part of this work for personal or classroom use is granted without fee provided that copies are not made or distributed for profit or commercial advantage and that copies bear this notice and the full citation on the first page. To copy otherwise, to republish, to post on servers or to redistribute to lists, requires prior specific permission and/or a fee.

Copyright 200X ACM X-XXXXX-XX-X/XX/XX ...\$10.00. tion assumes either small-signal ac noise or periodic stead-state noise analysis in a linear fashion, which cannot satisfy the need to verify the nonlinear transient noise analysis. The efficient numerical analysis of the transistor-level transient noise is required to facilitating the high-precision analog/RF designs in the nanometer region.

A number of previous arts have been proposed to address the aforementioned challenge when verifying the transient device noise Based on the Itô integral formulation, the transient noise can be estimated by solving the stochastic differential algebraic equations (SDAE) either under Euler-Maruyama or Milstein method $[2,3]$. One recent work in [4] has applied the stochastic integral scheme for SDAE, in particular stochastic analogues of the backward differentiation formula (BDF) and implicit trapezoidal rule (ITR) as in the traditional SPICE tools. However, this approach still requires the expensive Monte-Carlo iterations with the use of sampling-paths at each time point. Moreover, the expensive correlation analysis is required to calculate the noise variance. In addition, it is unknown how to model flicker noise inside this framework.

In $[5,6]$, a time-domain non-Monte-Carlo noise simulation considering thermal noise has been developed. Device noises are modeled as uncorrelated stochastic current sources. Since the magnitude of the noise in a signal is much smaller when compared to the magnitude of the signal itself, the solution of SDAE can be first piecewise-linearized along the nominal trajectory. The resulting reformulated SDAE is solved by the perturbation analysis. In order to calculate the noise variance, this approach also needs to perform the correlation analysis with intensive matrixoperations on the covariance matrix of circuit state variables at each time point, though the need of Monte Carlo iterations is avoided. The evaluation using the covariance matrix is expensive for the large-scale transient analysis. More importantly, because the perturbation analysis in $[5,6]$ is not applied to SDAE with Itô-integral form, the reformulation of SDAE under perturbation analysis might be inaccurate.

In this paper, we present an efficient and accurate non-MonteCarlo transient noise analysis to verify the stochastic noise of high-precision analog/RF circuits. First, it can model both thermal noise and flicker noise in the time domain by synthesized $\mathrm{RC}$ networks with white noise current sources, which can be connected to noise-free circuit elements. This lead to an Itô-integral formed SDAE with only Wiener processes. Next, in order to avoid inefficient Monte Carlo iterations and expensive co-variance matrix analysis $[4,5]$ when calculating the noise variance, we propose a one-time calculation with the use of stochastic orthogonal polynomials ( $\mathrm{SoPs}$ ). To the best of our knowledge, it is the first time to present the SoP solution for Itô integral based SDAE. Experiments show that SoPs based method is up to $488 \mathrm{X}$ faster than Monte Carlo method with similar accuracy. When compared with previous work, SoPs method can provide on average 6.8X speedup and higher accuracy. 
The rest of the paper is organized as following: we first review the background of the noise models and SoPs in Section 2. Then, we briefly summarize previous work in Section 3 and propose SoPs based method in Section 4. We show experimental results in Section 4 and conclude the paper in Section 5.

\section{BACKGROUND}

\subsection{Noise Models}

In our noise analysis, we consider white noise and flicker noise. Both thermal noise and shot noise are white noise and hence can be treated similarly. In this section, we briefly review the noise models.

\subsubsection{White Noise Model}

Both thermal noise and shot noise can be modeled as a white Gaussian noise current source that is connected to an ideal circuit element such as resistor or current source in parallel.

For instance, the thermal noise current for a resistor is

$$
i_{t h}(t)=\sqrt{\frac{2 k T}{R}} \xi(t)
$$

where $k$ is Boltzmann's constant, $T$ is the absolute temperature and $R$ is the resistance. $\xi(t)$ is a standard Gaussian white noise process, which is a stationary with a constant power spectral density (PSD) in frequency domain.

Similarly, there exists thermal noise in the channel of one MOS transistor associated with transconductance $g_{m}$, which can be modeled by:

$$
i_{t h}(t)=\sqrt{4 k T \theta \cdot g_{m}} \cdot \xi(t) .
$$

where $\theta$ depends on channel length and the operating region [7] which varys from $1 / 2$ to $2 / 3$.

\subsubsection{Flicker Noise Model}

Flicker noise is dominant in MOS transistors, which can be modeled by a noise current in parallel. Also, the PSD of flicker noise in MOS transistor can be generally written as

$$
S_{i}(f)=\frac{i_{f}^{2}}{\Delta f}=\frac{K_{F}}{C_{o x} W L} \times g_{m}^{2} \times \frac{1}{f}
$$

where $W$ is channel width, $L$ is channel length and $C_{o x}$ is gate oxide capacitance per unit area. Note that $K_{F}$ here is flicker noise coefficient, a constant depending on the process technology.

From equation (3), flicker noise has a time-varying PSD as a function of frequency, and thus it is a non-stationary noise process. That is why only white noise is included in the transient noise analysis for [4]. To include flicker noise during the transient noise analysis, we apply the synthesized $\mathrm{RC}$ circuit $[5,6]$ to generate the summation of Lorentzian spectra in (4) which approximates the $1 / \mathrm{f}$ noise PSD.

$$
S(f)=\frac{2 k T}{\pi C_{m}} \sum_{m=1}^{M} \frac{\varphi_{m}}{\varphi_{m}^{2}+f^{2}} \propto \frac{1}{f} .
$$

Here $\varphi_{m}$ is the pole-frequency and each Lorentzian spectra is represented by a white noise current source in parallel with an ideal group of resistor $R_{m}$ and capacitor $C_{m}$ shown in Fig(1).

In general, each flicker noise source can be represented by an ideal voltage-controlled current source, where the flicker noise current is $i(t)=g(t) \cdot v(t)$ with the output voltage $v(t)$ of one $R_{m}-C_{m}$ group circuit in Fig(1) and a time-varying transconductance $g(t)$. When all capacitors $C_{m}$ are fixed as constant value $C, g(t)$ can be written as

$$
g(t)=g_{m} \sqrt{\frac{K_{F}}{C_{o x} W L} \cdot \frac{\pi C}{2 k T}}
$$

Here, $g_{m}$ is the time-varying transconductance of MOS transistor. This important result shows that one can still model flicker noise by the synthesized RC network and white noise current source.

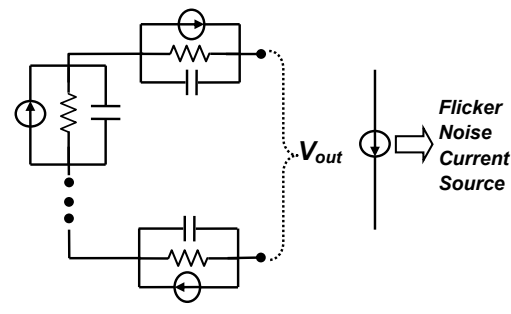

Figure 1: Flicker current noise source synthesis

\subsection{Stochastic Orthogonal Polynomial}

One recent advance in stochastic analysis is to apply stochastic orthogonal polynomial [8] or polynomial chaos to the nanometer scale integrated circuit analysis [9]. Based on the Askey scheme, any stochastic random variable can be represented by stochastic orthogonal polynomials (SoPs), and the random variable with different probability distribution type is associated with different type of SoP.

For example, for white noise current source with random variable $\xi$, its Gaussian distribution of $f(\psi)$ can be spanned by Hermite polynomials $\Phi(\psi)=\left[1, \psi, \psi^{2}-1, \cdots\right]^{T}$ as follows

$$
f(\psi)=\alpha_{0} \Phi_{0}+\alpha_{1} \Phi_{1}+\alpha_{2} \Phi_{2}+\cdots=\sum_{i=0}^{n} \alpha_{i} \Phi_{i}
$$

Note that SoPs satisfy the following orthogonal property under so-called point-collocation:

$$
\left\langle\Phi_{i}(\psi), \Phi_{j}(\psi)\right\rangle=\left\langle\Phi_{i}^{2}(\psi)\right\rangle \cdot \delta_{i j}
$$

where $\delta_{i j}$ is the Kronecker delta and $\langle *, *\rangle$ denotes an inner product.

As such, when the SoP representation is available, the mean and variance of $f(\psi)$ can be obtained from one-time calculation using collocation (up to the second order expansion) by:

$$
\begin{aligned}
& E(f(\psi))=\alpha_{0} \\
& \operatorname{Var}(f(\psi))=\alpha_{1}^{2}+2 \alpha_{2}^{2}
\end{aligned}
$$

In this paper, we show how to apply the SoP technique for the non-Monte-Carlo solution of the transient device noise analysis.

\section{PREVIOUS WORK}

One integrated circuit is composed of passive and active devices described by a number of terminal-branch equations. According to KCL's law, one can obtain a differential-algebraic equation (DAE) below

$$
A \frac{d}{d t} q(x(t))+f(x(t), t)=0 .
$$

Here, $x(t)$ is vector of state variables consisting of node voltages and branch currents. $q(x(t), t)$ contains charges and fluxes and $f(x(t), t)$ describes current-voltage relation. The constant matrix $A$ is incidence matrix determined by circuit topology.

\subsection{Itô Integral based SDAE}

When device noises become the interest, they can be modeled by noise current sources added to the deterministic DAE (9) by

$$
\underbrace{A \frac{d}{d t} q(x(t))+f(x(t), t)}_{\text {deterministic }}+\underbrace{\sum_{r=1}^{m} g_{r}(x(t), t) \xi_{r}(t)}_{\text {stochastic }}=0
$$

$g_{r}(x(t), t)$ is vector of noise intensities, and $\xi_{r}(t)$ is vector of noise sources (White noise). (10) is called stochastic differentialalgebraic equation (SDAE). Although (10) only differs from (9) by the stochastic noise sources, it requires a completely different numeric analysis. The primary difficulty to solve SDAE is that 
the required derivative of $x(t)$ is unavailable since $x(t)$ is nowhere differentiable due to the influence of stochastic noise sources.

Note that (10) can be interpreted as a stochastic Itô integral equation by integrating over one small time-interval $\left[t_{0}, t\right]$ :

$$
\left.A q(x(s))\right|_{t 0} ^{t}+\int_{t 0}^{t} f(x(s), s) d s+\sum_{r=1}^{m} \int_{t 0}^{t} g_{r}(x(t), t) d W_{r}(t)=0 .
$$

The second integral is called Itô Integral and thus equation (11) is called Itô Integral based $S D A E[10,4] . W_{r}(t)$ denotes the Brownian motion or the Wiener Process, obtained by integrating the white noise: $W_{r}(t)=\int_{0}^{t} \xi_{r}(s) d s=\int_{0}^{t} d W_{r}(s)$. One Wiener process is characterized by the initial value $W(0)=0$ and the independent non-overlapping increments $\Delta W\left(t_{n}\right)=W\left(t_{n}\right)-$ $W\left(t_{n-1}\right) \sim N\left(0, h_{n}\right)$. Here, $h_{n}=t_{n}-t_{n-1}$ is the integration time-step.

Under the the form of Itô Integral based SDAE, the work in [10] proved the existence and uniqueness of the solution. The work in [4] further derived several stochastic integration methods. For example, one stochastic two-step backward differentiation formula $\left(\mathrm{BDF}_{2}\right)$-Maruyama method based discretization of (11) becomes

$$
\begin{aligned}
& A \frac{q\left(x_{n}\right)-\frac{4}{3} q\left(x_{n-1}\right)+\frac{1}{3} q\left(x_{n-2}\right)}{h}+\frac{2}{3} f\left(x_{n}\right)+ \\
& \sum_{r=1}^{m} g_{r}\left(x_{n-1}\right) \frac{\Delta W_{n}^{r}}{h}-\frac{1}{3} \sum_{r=1}^{m} g_{r}\left(x_{n-2}\right) \frac{\Delta W_{n-1}^{r}}{h}=0
\end{aligned}
$$

At each time step, (12) can be solved by Newton method with a number of Monte Carlo based sampling-paths for the Wiener process $\Delta W_{r}$. The noise variance is calculated afterward at each time-step with Monte Carlo iterations. This is the key idea for the transient noise analysis in $[4,10]$. The limitation of this approach is the inefficiency due to the Monte Carlo iterations where the complexity increases with the number of noise sources and the scale of circuit.

\subsection{Perturbation based SDAE}

Considering that the magnitude of noises $(-100 \mathrm{db})$ is much smaller than the magnitude of signals, it is accurate to solve SDAE for transient noise application by the perturbation analysis from $[5,6]$. One can first obtain the nominal transient trajectory or solution $x^{(0)}(t)$ for (9). The SDAE in (10) is then piecewiselinearized

$$
\begin{aligned}
& A\left[\frac{d}{d} q\left(x^{(0)}(t)\right)+\left.\frac{\partial q(x(t))}{\partial x}\right|_{x=x^{(0)}} \cdot\left(\dot{x}(t)-\dot{x}^{(0)}(t)\right)\right] \\
& +\left[f\left(x^{(0)}(t), t\right)+\left.\frac{\partial f(x(t))}{\partial x}\right|_{x=x^{(0)}} \cdot\left(x(t)-x^{(0)}(t)\right)\right] \\
& +\sum_{r=1}^{m} g_{r}\left(x^{(0)}(t), t\right) \xi_{r}(t)=0
\end{aligned}
$$

Based on the nominal solution of (9). For the simplicity of notation, one can define

$$
\begin{aligned}
& C^{(0)}(t)=\left.\frac{\partial q(x(t))}{\partial x}\right|_{x=x}, G^{(0)}(t)=\left.\frac{\partial f(x(t))}{\partial x}\right|_{x=x^{(0)}} \\
& \Delta x=x(t)-x^{(0)}(t), \Delta \dot{x}=\dot{x}(t)-\dot{x}^{(0)}(t) \\
& F=\sum_{r=1}^{m} g_{r}(x(t), t) .
\end{aligned}
$$

As such, the linearized SDAE is simplified as

$$
A \cdot C^{(0)}(t) \cdot \Delta \dot{x}+G^{(0)}(t) \cdot \Delta x+F \cdot \xi(t)=0 .
$$

Since $C^{(0)}(t)$ may have zero columns, [5] reordered variables $\Delta x$ so that zero columns of $C^{(0)}(t)$ are grouped at the right-hand side of matrix. Hence, (15) becomes:

$$
\begin{aligned}
& {\left[\begin{array}{cc}
C_{11}(t) & 0 \\
0 & 0
\end{array}\right]\left[\begin{array}{c}
\Delta \dot{x}_{1} \\
\Delta \dot{x}_{2}
\end{array}\right]+\left[\begin{array}{ll}
G_{11}(t) & G_{12}(t) \\
G_{21}(t) & G_{22}(t)
\end{array}\right]\left[\begin{array}{c}
\Delta x_{1} \\
\Delta x_{2}
\end{array}\right]} \\
& +\left[\begin{array}{c}
F_{1}(t) \\
F_{2}(t)
\end{array}\right] \xi=0
\end{aligned}
$$

where $C_{11}(t)$ consists of non-zero columns of $A \cdot C^{(0)}(t)$. This results in an inherent stochastic differential equation (SDE) with one additional algebraic constraint by

$$
\begin{aligned}
& G_{11}(t) \Delta x_{1}+G_{12}(t) \Delta x_{2}+C_{11}(t) \Delta \dot{x}_{1}+F_{1}(t) \xi=0 \\
& G_{21}(t) \Delta x_{1}+G_{22}(t) \Delta x_{2}+F_{2}(t) \xi=0 \\
& \Delta x=\left[\begin{array}{ll}
\Delta x_{1} & \Delta x_{2}
\end{array}\right]^{T}
\end{aligned}
$$

The first equation in (17) owns the standard SDE form.

Instead of performing Monte Carlo iterations to calculate the noise variance, $[5,6]$ applied one non-Monte-Carlo approach to calculate the noise variance from the covariance matrix. Based on the Itô theorem[11], the covariance matrix $K_{1}(t)$ for the SDE in (17) can be expressed in the differential Lyapunov matrix equation by

$$
\begin{aligned}
& \dot{K}_{1}(t)=-\left(G_{11}(t)+G_{12}(t) \cdot\left(-\left(G_{22}(t)\right)^{-1} G_{21}(t)\right)\right) K_{1}(t) \\
& +K_{1}(t)\left[-\left(G_{11}(t)+G_{12}(t) \cdot\left(-\left(G_{22}(t)\right)^{-1} G_{21}(t)\right)\right)\right]^{T} \\
& +\left[-\left(F_{1}(t)+G_{12}(t) \cdot\left(-\left(G_{22}(t)\right)^{-1} F_{2}(t)\right)\right)\right] \\
& \cdot\left[-\left(F_{1}(t)+G_{12}(t) \cdot\left(-\left(G_{22}(t)\right)^{-1} F_{2}(t)\right)\right)\right]^{T}
\end{aligned}
$$

As such, the covariance matrix $K_{1}\left(t_{n}\right)$ can be obtained from the above correlation analysis at time step $t_{n}$, and variances of circuit variables at $t_{n}$ can be further extracted from the diagonal elements of $K_{1}\left(t_{n}\right)$. Though this method avoids massive samplings and iterations from Monte Carlo, there is a number of time-consuming operations to solve the inherent SDE numerically and to perform the operations on the Lyapunov matrix.

\section{SOP BASED NMC TRANSIENT NOISE ANALYSIS}

As discussed in Section 3, the primary limitation of the current transient noise analysis is lack of the efficiency. Moreover, a complete transient noise solution needs to deal with not only white noise but also flicker noise. Applying stochastic orthogonal polynomials (SoPs) to obtain the mean and variance by one-time calculation, we develop the non-Monte-Carlo numerical analysis in this section.

\subsection{Itô Integral based SDAE with Flicker Noise}

To include flicker noise into the Itô integral based SDAE in (10), we denote the synthesized RC network for flicker noise as synthesized circuit. There are two equivalent approaches to calculate the contribution of flicker noise:

- Static Method: Flicker noise is computed beforehand by performing the transient noise analysis on the synthesized circuit using (12). Afterward, the flicker noise is injected into the original circuit later for the total transient noise analysis.

- Dynamic Method: The original circuit is first augmented with the corresponding synthesized circuits. Then, the transient noise analysis is performed on the augmented circuit using (12).

The dynamic method requires to create extra nodes for the synthesized circuit and thus increases the complexity. In this paper, we take the static method as an example for the illustration, but the proposed SoP techniques can also be applied to the case of dynamic method in a similar fashion.

Let $i_{f}^{k}(t)$ be the value of $k$-th white noise current source for the flicker noise. Since flicker noises can be modeled as addictive noise current sources, (10) becomes

$\underbrace{A \frac{d}{d t} q(x(t))+f(x(t), t)}_{\text {noise free }}+\underbrace{\sum_{k=1}^{n} T_{k} \cdot i_{f}^{k}(t)}_{\text {flicker noise }}+\underbrace{\sum_{r=1}^{m} g_{r}(x(t), t) \xi_{r}(t)}_{\text {thermal noise }}=0$ 
where $T_{k}$ is topology matrix determining how to connect flicker noise current sources into the circuit.

Similarly, in order to obtain the Itô integral based SDAE with flick noise, (19) can be integrated over the time-interval and becomes

$$
\begin{aligned}
& \left.A q(x(s))\right|_{t 0} ^{t}+\int_{t 0}^{t} f(x(s), s) d s+\sum_{k} \int_{t 0}^{t} T_{k} \cdot i_{f}^{k}(t) \cdot d s \\
& +\sum_{r=1}^{m} \int_{t 0}^{t} g_{r}(X(t), t) d W_{r}(t)=0
\end{aligned}
$$

The corresponding $\mathrm{BDF}_{2}$-Maruyama method with only increments of Wiener process at $n$-th discretized time instant is derived by

$$
\begin{aligned}
& A \frac{q\left(x_{n}\right)-\frac{4}{3} q\left(x_{n-1}\right)+\frac{1}{3} q\left(x_{n-2}\right)}{h}+\frac{2}{3} f\left(x_{n}\right) \\
& +\frac{1}{2} \sum_{k} T_{k} \cdot i_{f}^{k}\left(t_{n}\right)+\sum_{r=1}^{m} g_{r}\left(x_{n-1}\right) \frac{\Delta W_{n}^{r}}{h} \\
& -\frac{1}{3} \sum_{r=1}^{m} g_{r}\left(x_{n-2}\right) \frac{\Delta W_{n-1}^{r}}{h}=0
\end{aligned}
$$

Table 1: SoP Expansions for Random Variables.

\begin{tabular}{c|c|c}
\hline \multicolumn{2}{c|}{ Random Variables } & SoP Expansion \\
\hline known & $i_{f}^{k}\left(t_{n}\right)$ & $g\left(t_{n}\right) \cdot\left(\gamma_{0}^{k}\left(t_{n}\right) \Phi_{0}+\gamma_{1}^{k}\left(t_{n}\right) \Phi_{1}+\cdots\right)$ \\
\cline { 2 - 3 } variables & $\Delta W_{n}^{r}$ & $\alpha_{0}^{r}\left(t_{n}\right) \Phi_{0}+\alpha_{1}^{r}\left(t_{n}\right) \Phi_{1}+\cdots$ \\
\hline \multirow{3}{*}{$\begin{array}{c}\text { unknown } \\
\text { variables }\end{array}$} & $q\left(x_{n}\right)$ & $q\left(x_{n}^{(0)}\right)+C_{n}^{(0)} \cdot\left(\beta_{1}\left(t_{n}\right) \Phi_{1}+\cdots\right)$ \\
\cline { 2 - 3 } & $f\left(x_{n}\right)$ & $f\left(x_{n}^{(0)}\right)+G_{n}^{(0)} \cdot\left(\beta_{1}\left(t_{n}\right) \Phi_{1}+\cdots\right)$ \\
\cline { 2 - 3 } & $x_{n}$ & $x_{n}^{(0)} \Phi_{0}+\beta_{1}\left(t_{n}\right) \Phi_{1}+\cdots$ \\
\hline
\end{tabular}

\subsection{SoP Collocation of Itô Integral based SDAE}

The above equation (21) and (12) can be solved with Monte Carlo iterations, which is very inefficient. Therefore, we develop one efficient Non-Monte-Carlo (NMC) transient noise analysis using the stochastic orthogonal polynomials (SoPs). This leads to one-time calculation of the noise variance. We will discuss how to represent the random variables in $(21)$ by SoPs and further solve $x_{n}$ by collocation. Note that the random variables in this section include $q\left(x_{n}\right), f\left(x_{n}\right), \Delta W_{n}^{r}, i_{f}^{k}(t)$ and $x_{n}$. In the following, we show derivations of their SoP representations one by one, respectively. The results of SoP expansions are summarized in Table 1 .

\subsubsection{SoP Expansions of $q\left(x_{n}\right)$ and $f\left(x_{n}\right)$}

The magnitudes of both white noise and flicker are much smaller than the one of signal, therefore, one can first obtain the nominal transient solution $x_{n}^{(0)}$. And accordingly, $q\left(x_{n}^{(0)}\right)$ and $f\left(x_{n}^{(0)}\right)$. Along this nominal transient trajectory, $x_{n}, q\left(x_{n}\right)$ and $f\left(x_{n}\right)$ can be further piecewise-linearized by

$$
\begin{aligned}
& x_{n}=x_{n}^{(0)}+\Delta x_{n} \\
& q\left(x_{n}\right)=q\left(x_{n}^{(0)}\right)+\left.\frac{\partial q}{\partial x}\right|_{x=x_{n}^{(0)}} \cdot \Delta x_{n} \\
& f\left(x_{n}\right)=f\left(x_{n}^{(0)}\right)+\left.\frac{\partial f}{\partial x}\right|_{x=x_{n}^{(0)}} \cdot \Delta x_{n} .
\end{aligned}
$$

Therefore, one only needs to further solve $\Delta x_{n}=x_{n}-x_{n}^{(0)}$ instead of $x_{n}$.

Note that $\Delta x_{n}$ is the stochastic perturbation to $x_{n}^{(0)}$ with the Gaussian distribution. Therefore, the noise mean is $E\left(\Delta x_{n}\right)=$ 0 and hence $E\left(x_{n}\right)=x_{n}^{(0)}$. Moreover, the noise variance is
$\operatorname{Var}\left(x_{n}\right)=\operatorname{Var}\left(\Delta x_{n}\right)$, which leads to the SoP expansions of $x_{n}$ and $\Delta x_{n}$ by

$$
\begin{aligned}
& \Delta x_{n}=0 \cdot \Phi_{0}+\beta_{1}\left(t_{n}\right) \Phi_{1}+\cdots \\
& x_{n}=x_{n}^{(0)}+\Delta x_{n}=x_{n}^{(0)} \Phi_{0}+\beta_{1}\left(t_{n}\right) \Phi_{1}+\cdots
\end{aligned}
$$

Accordingly, one can obtain the SoP expansions of $q\left(x_{n}\right)$ and $f\left(x_{n}\right)$ by

$$
\begin{aligned}
& q\left(x_{n}\right)=q\left(x_{n}^{(0)}\right)+C_{n}^{(0)} \cdot\left(\beta_{1}\left(t_{n}\right) \Phi_{1}+\cdots\right) \\
& f\left(x_{n}\right)=f\left(x_{n}^{(0)}\right)+G_{n}^{(0)} \cdot\left(\beta_{1}\left(t_{n}\right) \Phi_{1}+\cdots\right)
\end{aligned}
$$

Here, the capacitive and conductive Jacobians are used for the simplicity of notation

$$
C_{n}^{(0)}=\left.\frac{\partial q}{\partial x}\right|_{x=x_{n}^{(0)}} ; G_{n}^{(0)}=\left.\frac{\partial f}{\partial x}\right|_{x=x_{n}^{(0)}} .
$$

\subsubsection{SoP Expansion of $\Delta W_{n}^{r}$ and $i_{f}^{k}\left(t_{n}\right)$}

The increments of Wiener process $\Delta W_{n}^{r} \sim N\left(0, h_{n}\right)$ can be represented by SoPs as

$$
\Delta W_{n}^{r}=\alpha_{0}^{r}\left(t_{n}\right) \Phi_{0}+\alpha_{1}^{r}\left(t_{n}\right) \Phi_{1}+\alpha_{2}^{r}\left(t_{n}\right) \Phi_{2}+\cdots
$$

With techniques in [9], $\alpha_{i}^{r}\left(t_{n}\right)$ can be obtained with known distribution of $\Delta W_{n}^{r}$. Take the first order expansion as an example, $\alpha_{0}^{r}\left(t_{n}\right)=E\left(\Delta W_{n}^{r}\right)=0$, and $\left(\alpha_{1}^{r}\left(t_{n}\right)\right)^{2}=\operatorname{Var}\left(\Delta W_{n}^{r}\right)=h_{n}$

In addition, the SoP expansion of $k$-th flicker noise current source $i_{f}^{k}\left(t_{n}\right)$ becomes

$$
i_{f}^{k}\left(t_{n}\right)=g\left(t_{n}\right) \cdot v\left(t_{n}\right)=g\left(t_{n}\right) \cdot\left[\gamma_{0}^{k}\left(t_{n}\right) \Phi_{0}+\gamma_{1}^{k}\left(t_{n}\right) \Phi_{1}+\cdots\right] .
$$

Here, $g(t)$ is the transconductance defined in (5). $v(t)$ is the output voltage of flicker noise synthesized circuit in Fig.(1), which only contains thermal noises and can be solved with $\mathrm{BDF}_{2}$-Maruyama method in (12) under the scheme of static method.

\subsubsection{Solution of $\gamma_{i}$ by SoP Collocation}

Because the static method is considered to calculate the contribution of flicker noise, $\left\{\gamma_{i}\right\}$ needs to be first determined by SoP collocation. By expanding $q\left(x_{n}\right), f\left(x_{n}\right)$ and $\Delta W_{n}^{r}$ via SoPs for the synthesized circuit, one obtain a new SDAE under BDF $2^{-}$ Maruyama discretization described in (27). By applying the innerproduct with $\Phi_{i}(i=0,1, \cdots)$ to $(27)$, one can obtain a set of equations corresponding to the order of SoP for $\gamma_{i}\left(t_{n}\right)$. For example, the resulting $\Phi_{1}$ becomes

$$
\begin{aligned}
& A \frac{C_{n}^{(0)} \cdot \gamma_{1}\left(t_{n}\right)-\frac{4}{3} C_{n-1}^{(0)} \cdot \gamma_{1}\left(t_{n-1}\right)+\frac{1}{3} C_{n-2}^{(0)} \cdot \gamma_{1}\left(t_{n-2}\right)}{h} \\
& +\left(\frac{2}{3} G_{n}^{(0)} \cdot \gamma_{1}\left(t_{n}\right)\right)+\frac{2}{3} \sum_{r=1}^{m} g_{r} \cdot \frac{\alpha_{1}\left(t_{n}\right)}{h}=0
\end{aligned}
$$

As a result, $\gamma_{i}\left(t_{n}\right)$ can be solved from (28).

\subsubsection{Solution of $x_{n}$ by SoP Collocation}

When the contribution from the flicker noise is obtained, one can further obtain the total transient noise by one more SoP collocation. By applying the inner-product with $\Phi_{i}(i=0,1, \cdots)$ to (21), coefficients $\beta_{i}$ of SoP expansion of $x_{n}$ in (23) can be computed. For instance, the equation corresponding to $\Phi_{1}$ is

$$
\begin{aligned}
& A \frac{C_{n}^{(0)} \cdot \beta_{1}\left(t_{n}\right)-\frac{4}{3} C_{n-1}^{(0)} \cdot \beta_{1}\left(t_{n-1}\right)+\frac{1}{3} C_{n-2}^{(0)} \cdot \beta_{1}\left(t_{n-2}\right)}{h} \\
& +\left(\frac{2}{3} G_{n}^{(0)} \cdot \beta_{1}\left(t_{n}\right)\right)+\frac{1}{2} \sum_{k} T_{k} \cdot g_{k}\left(t_{n}\right) \cdot \gamma_{1}^{k}\left(t_{n}\right) \\
& +\frac{2}{3} \sum_{r=1}^{m} g_{r} \cdot \frac{\alpha_{1}\left(t_{n}\right)}{h}=0
\end{aligned}
$$

The above equation can be solved for $\beta_{1}\left(t_{n}\right)$ at $n$-th time instant and is repeatedly solved for all time instants. As a result, the $\beta_{i}$ can be calculated as a function of time. Therefore, the noise variance at $t_{n}$ is efficiently obtained by $\operatorname{Var}\left(x_{n}\right)=\left\{\beta_{1}\left(t_{n}\right)\right\}^{2}$. 


$$
\begin{aligned}
A \cdot \frac{\left(q\left(x_{n}^{(0)}\right)+C_{n}^{(0)} \cdot \sum_{i=0}^{n} \gamma_{i}\left(t_{n}\right) \Phi_{i}\right)}{h} & -A \cdot \frac{4}{3} \frac{\left(q\left(x_{n-1}^{(0)}\right)+C_{n-1}^{(0)} \cdot \sum_{i=0}^{n} \gamma_{i}\left(t_{n-1}\right) \Phi_{i}\right)}{h}+A \cdot \frac{1}{3} \frac{\left(q\left(x_{n-2}^{(0)}\right)+C_{n-2}^{(0)} \cdot \sum_{i=0}^{n} \gamma_{i}\left(t_{n-2}\right) \Phi_{i}\right)}{h} \\
& +\left(\frac{2}{3}\left(f\left(x_{n}^{(0)}\right)+G_{n}^{(0)} \cdot \sum_{i=0}^{n} \gamma_{i}\left(t_{n}\right) \Phi_{i}\right)\right)+\frac{2}{3} \sum_{r=1}^{m} g_{r} \frac{\sum_{i=0}^{n} \alpha_{i}\left(t_{n}\right) \Phi_{i}}{h}=0
\end{aligned}
$$

\section{EXPERIMENTAL RESULTS}

We have implemented the proposed non-Monte-Carlo transient noise analysis in a Matlab-based circuit simulator, and all experiments are carried out on a Linux server with a $2.4 \mathrm{GHz}$ Xeon processor and 4GB memory. We use SoP method for two purposes: SoP expansion of flicker noise validated in Section 5.1, and the SoP expansion for transient noise analysis verified in Section 5.2. Two analogue circuits including a CMOS comparator and a 3-stage ring oscillator are used compare SoP method with Monte Carlo and Perturbation based SDAE analysis [5]. For all circuits in Section 5.2, we include both thermal noise and flicker noise sources in our simulation.

\subsection{SoP Expansion to Model Flicker Noise}

SoP expansion of flicker $(1 / \mathrm{f})$ noise is validated by standard deviation of $1 / \mathrm{f}$ noise $\left(\sigma_{\text {noise }}\right)$ in the time domain. Using the $1 / \mathrm{f}$ noise synthesized circuit in Fig.(1), we perform both 1000 Monte Carlo simulations and SoP method to generate $1 / \mathrm{f}$ noise $\sigma_{\text {noise }}$ as a function of time. The speedup of SoP method over Monte Carlo simulations is $1030.85(s) / 1.01(s) \approx 1020 X$.

Fig. (2) shows the $\sigma_{\text {noise }}$ from both methods. Initially, there are large differences, but error reduces as the time increases. Note that the difference at initial stage is not important since simulation time of flicker noise is very long. In fact, the average error of SoP method with respect to Monte Carlo is $0.32 \%$. Therefore, the accuracy of SoP method is satisfied. In the following experiments, we use the SoP expansion of flicker noise.

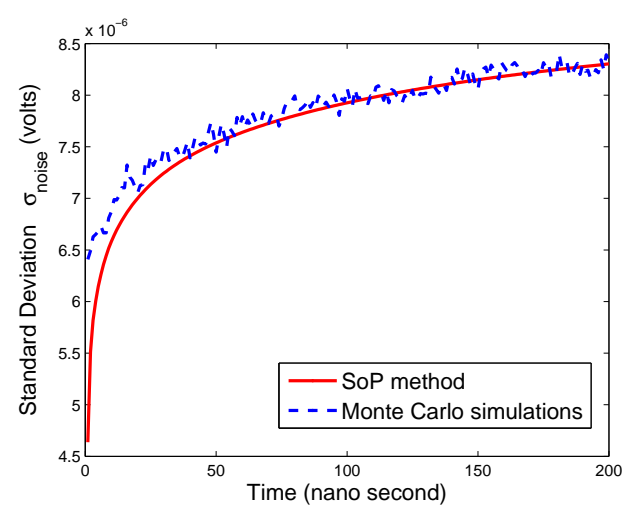

Figure 2: $\sigma_{\text {noise }}$ comparison for the flicker (1/f) noise

\subsection{SoP Method for Transient Noise Analysis}

\subsubsection{Accuracy for CMOS comparator}

The first example is one CMOS comparator as shown in Fig.(3) and detailed information about post layout of this circuit is illustrated in Table.(2). We consider both thermal noise and flicker noise to all eight MOSFETs, while only thermal noise is considered for all resistors.

First, we run Monte Carlo simulations to capture the standard deviation $\left(\sigma_{\text {output }}\right)$ of impacted output voltage with $0.5 \%$ error in the time domain. It is denoted as one red dash line with plus signs

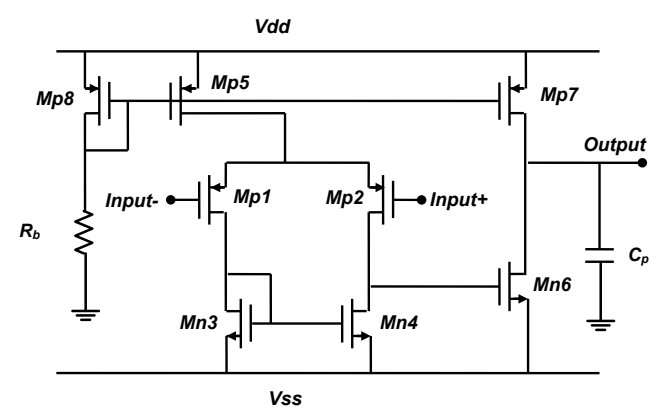

Figure 3: A CMOS comparator implementation with PMOS input drivers

in Fig.(4). Then, perturbation based SDAE analysis is conducted for $\sigma_{\text {output }}$ which leads to a black dash line with cross signs. In addition, the $\sigma_{\text {output }}$ from SoP method is marked by a blue dash line with triangle sign.

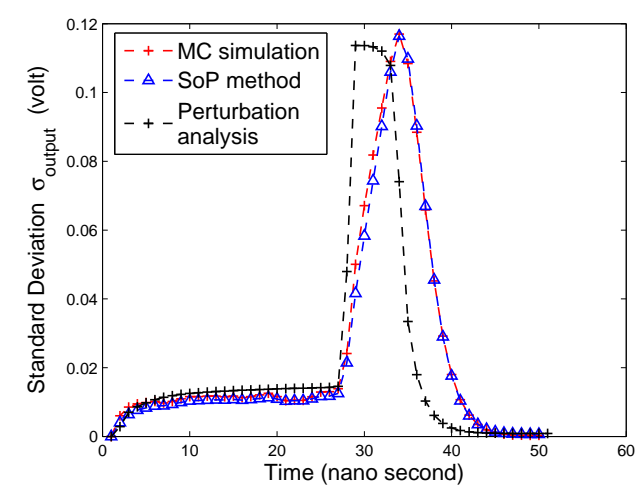

Figure 4: Comparison of $\sigma_{\text {output }}$ for comparator

From the comparison in Fig. (4), SoP method fits with Monte Carlo simulations very well in the time domain, while perturbation based SDAE analysis[5] fails to keep high accuracy in the pulse area. This shows that SoP method can provide higher accuracy. Also, the total CPU runtime are shown in Table.(3).

\subsubsection{Accuracy for 3-stage CMOS ring-oscillator}

We further consider a 3-stage ring-oscillator as shown in Fig.(5) and post layout details is list in Table.(2). Similarly, we study the standard deviation of output voltage $\sigma_{\text {output }}$ in this example.

We first introduce thermal noises and $1 / \mathrm{f}$ noises to all MOSFETs and run Monte Carlo simulations to obtain $\sigma_{\text {output }}$ with $0.5 \%$ error, which is plotted as a red dash line with plus signs in Fig.(6). Note that $\sigma_{\text {output }}$ is not constant as a function of time, because $1 / \mathrm{f}$ noise is one non-stationary random processes and its PSD is not a constant in frequency domain.

Then, perturbation based SDAE analysis is performed to gen- 
Table 2: Circuit Information for Different Examples.

\begin{tabular}{l|c|c|c|c}
\hline circuit example & \#nodes & \#devices & $\begin{array}{c}\text { \#thermal } \\
\text { noise }\end{array}$ & $\begin{array}{c}\text { \#flicker } \\
\text { noise }\end{array}$ \\
\hline CMOS Invertor & 13 & 21 & 10 & 1 \\
\hline OPAM & 46 & 61 & 43 & 8 \\
\hline Comparator & 41 & 54 & 37 & 8 \\
\hline Oscillator & 37 & 57 & 37 & 6 \\
\hline
\end{tabular}

erate $\sigma_{\text {output }}$ as one black dash line with cross signs. Additionally, proposed SoP method computes $\sigma_{\text {output }}$ denoted by one blue dash line with triangle signs that visually identical to those from Monte Carlo simulation.

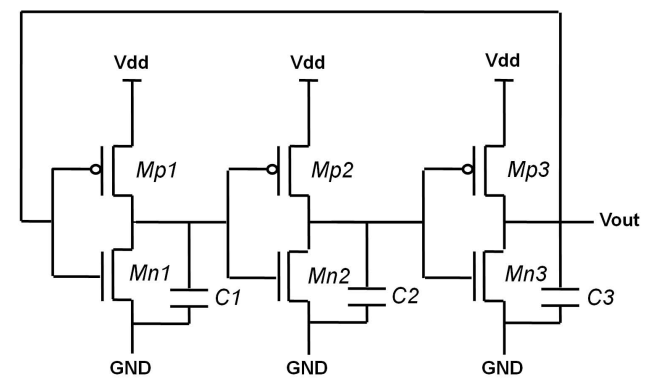

Figure 5: A 3-stage CMOS ring-oscillator

As shown in Fig.(6), perturbation based SDAE analysis can provide satisfied accuracy during low-to-high and high-to-low transitions, but fails in the peak regions. In contrast, SoP method is able to remain very high accuracy within the entire time domain.

Table 3: Accuracy and Total Runtime Comparison for Different Circuit Examples. (Time Unit: second)

\begin{tabular}{c|c|c|c|c|c}
\hline & & Invertor & OPAM & Comparator & Oscillator \\
\hline \multirow{2}{*}{$\begin{array}{c}\text { MC } \\
\text { method }\end{array}$} & error & $0.5 \%$ & $0.5 \%$ & $0.5 \%$ & $0.5 \%$ \\
\cline { 2 - 6 } & time & 91.95 & 4266.64 & 2226.71 & 146851.2 \\
\cline { 2 - 6 } & speedup & $1 \mathrm{X}$ & $1 \mathrm{X}$ & $1 \mathrm{X}$ & $1 \mathrm{X}$ \\
\hline \multirow{2}{*}{$\begin{array}{c}\text { Pert. } \\
\text { analysis }\end{array}$} & error & $5.24 \%$ & $18.6 \%$ & $36.4 \%$ & $33.7 \%$ \\
\cline { 2 - 6 } & time & 1.84 & 54.71 & 12.56 & 304.3 \\
\cline { 2 - 6 } & speedup & $50 \mathrm{X}$ & $78 \mathrm{X}$ & $177 \mathrm{X}$ & $483 \mathrm{X}$ \\
\hline \multirow{2}{*}{$\begin{array}{c}\text { SoP } \\
\text { method }\end{array}$} & error & $0.43 \%$ & $0.93 \%$ & $1.78 \%$ & $1.62 \%$ \\
\cline { 2 - 6 } & time & 1.87 & 52.35 & 12.72 & 300.91 \\
\cline { 2 - 6 } & speedup & $49 \mathrm{X}$ & $81.5 \mathrm{X}$ & $175 \mathrm{X}$ & $488 \mathrm{X}$ \\
\hline
\end{tabular}

${ }^{1}$ Perturbation based SDAE analysis

In fact, the accuracy of all methods are compared in Table.(3), Monte Carlo simulation can provide high accuracy ( $0.5 \%$ error), and perturbation based SDAE analysis involves large error (up to $36 \%$ accuracy loss). SoP method can achieve the similar accuracy around $1 \%$ as Monte Carlo.

\subsubsection{Runtime Comparison}

From Table.(3), Monte Carlo method is inefficient and requires a huge amount of computation time. In addition, perturbation based SDAE analysis and SoP method have similar efficiency which are up to $488 \mathrm{X}$ faster than Monte Carlo method, but SoP method can provide much higher accuracy.

Note that the total runtime for both NMC methods in Table.(3) contains both nominal transient simulation and standard deviation $\sigma_{\text {output }}$ computation, where the nominal transient analysis dominates the total runtime. We further compare the runtime of $\sigma_{\text {output }}$ computation for both NMC methods in Table(4). As shown in the table, SoP method is around $6.8 X$ faster than perturbation based SDAE analysis[5], and this speed advantage is expected to be bigger when scale of circuits increases.

\section{CONCLUSION}

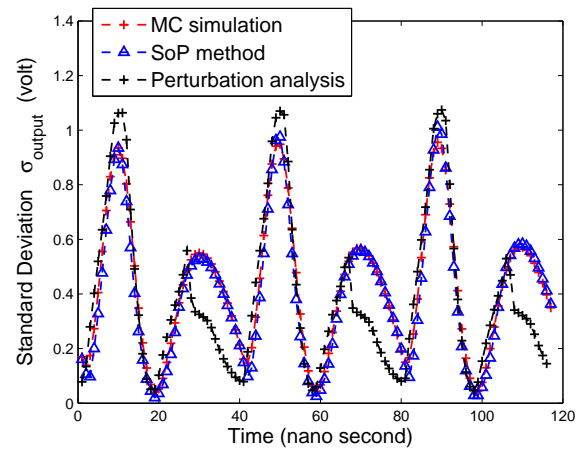

Figure 6: Comparison of $\sigma_{\text {output }}$ for Oscillator

In this paper, Itô integral based stochastic differential algebra equation (SDAE) is deployed to consider both white and flicker noise for high precision analog/RF circuits. One non-Monte-Carlo solution is developed based on the stochastic orthogonal polynomial (SoP) collocation to solve the piecewise-linearized SDAE. The noise variance can be obtained by just one-time calculation at each time-point. Extensive experiments demonstrated that proposed method is up to $488 \mathrm{X}$ faster than Monte Carlo method with a similar accuracy, and achieves on average $6.8 \mathrm{X}$ speedup over existing non-Monte-Carlo method.

Table 4: Runtime for $\sigma_{\text {output }}$ computation.

\begin{tabular}{l|c|c|c|c}
\hline \multirow{2}{*}{ circuit example } & \multicolumn{2}{c|}{ Perturbation Analysis } & \multicolumn{2}{c}{ SoP Method } \\
\cline { 2 - 5 } & time (s) & speedup & time $(\mathrm{s})$ & speedup \\
\hline CMOS Invertor & 0.06 & $1 \mathrm{X}$ & 0.013 & $4.6 \mathrm{X}$ \\
\hline OPAM & 3.07 & $1 \mathrm{X}$ & 0.41 & $7.5 \mathrm{X}$ \\
\hline Comparator & 1.36 & $1 \mathrm{X}$ & 0.168 & $8.1 \mathrm{X}$ \\
\hline Oscillator & 5.46 & $1 \mathrm{X}$ & 0.8 & $6.8 \mathrm{X}$ \\
\hline
\end{tabular}

\section{REFERENCES}

A. Mehrotra, "Noise analysis of phase-locked loops," in Proc. IEEE/ACM Int. Conf. Computer-aided-design (ICCAD), pp. 277-282, 2000.

[2] G. N. Milstein, "Numerical integration of stochastic differential equations," 2005.

[3] P. E. Kloeden and E. Platen, "Numerical solution of stochastic differential equations," 1999.

[4] G. Denk, W. Romisch, T. Sickenberger, and R. Winkler, "Efficient transient noise analysis in circuit simulation," Proceedings in Applied Mathematics and Mechanics, vol. 6, pp. 55-58, 2006.

[5] A. Demir, E. W. Y. Liu, and A. L. Sangiovanni-Vincentelli, "Time-domain non-Monte Carlo noise simulation for nonlinear dynamic circuits with arbitrary excitations," in Proc. IEEE/ACM Int. Conf. Computer-aided-design (ICCAD), pp. 598-603, 1994.

[6] A. Demir, E. Liu, and A. Sangiovanni-Vincentelli, "Time-domain non-Monte Carlo noise simulation for nonlinear dynamic circuits with arbitrary excitations," Computer-Aided Design of Integrated Circuits and Systems, IEEE Transactions on, vol. 15, no. 5, pp. $493-505,1996$

[7] Y. Cheng, M. Chan, K. Hui, M. Jeng, Z. Liu, J. Huang, K. Chen, J. Chen, R. Tu, P. K. Ko, and C. Hu, "BSIM3v3 manual," Univ. California at Berkeley, 1996.

[8] S. Vrudhula, J. Wang, and P. Ghanta, "Hermite polynomial based interconnect analysis in the presence of process variations," IEEE Tran. on Computer-aided-design (TCAD), vol. 25 , no. 10 , pp. 2001-2011, 2006.

[9] D. Xiu and G. E. Karniadakis, "The Wiener-Askey polynomial chaos for stochastic differential equations," SIAM J. Sci. Comput., vol. 24, pp. 619-644, 2002.

[10] R. Winkler, "Stochastic differential algebraic equations of index-1 and applications in circuit simulation," J. Comput. Appl. Math., vol. 163, pp. 435-463, 2004.

[11] A. L., "Stochastic differential equations: Theory and applications," 1974. 